Hugo Spinelli ${ }^{1}$ Marcio Alazraqui ${ }^{1}$ Diego Galeano ${ }^{1}$ Sabrina Calandrón ${ }^{2}$

\section{Muertes por violencias en Argentina: dos estudios de caso en los Municipios de Venado Tuerto y San Rafael}

\author{
Violence-related deaths in Argentina: \\ two case studies in the cities of Venado Tuerto and San Rafael
}

${ }^{1}$ Instituto de Salud Colectiva, Universidad Nacional de Lanús (UNLa). Av. 29 de Septiembre 3901, edificio "Leonardo Werthein" (1826) Remedios de Escalada. Lanús Provincia de Buenos Aires Argentina.

hugospinelli09@gmail.com ${ }^{2}$ Comisión Nacional de Investigaciones Científicas y Técnicas (CONICET).

\begin{abstract}
This article presents the results of a comparative study of two Argentinian cities, namely Venado Tuerto and San Rafael, which revealed different trends in the rates of firearm-related homicides. The methodology combined two strategies of analysis: semi-structured interviews with key informants (municipal and provincial government agents in different areas of management, as well as members of non-governmental organizations) and focus groups with actors involved in medical care, education, and religious institutions. The results suggest little difference between cities in which rates have increased and those in which rates have decreased. The most significant difference was that in Venado Tuerto a greater fragility of public institutions was observed due to the lack of articulation between such institutions. In San Rafael, the actors interviewed attribute the low level of conflict to a violence prevention network in which provincial and municipal agencies interact. Although neither city is violent at the most critical Latin American levels, the different results shown in Venado Tuerco and San Rafael indicate the possibility of bringing institutions together in a joint framework of conversations, agreements and policies.
\end{abstract}

Key words Homicide, Violence, Mortality, Firearms, Local Government, Argentina
Resumen Este artículo ofrece los resultados de un estudio comparado de dos municipios: Venado Tuerto y San Rafael de la República Argentina, que presentaron tendencias diferentes en relación a las tasas de homicidios por armas de fuego. La metodología combinó dos estrategias de análisis: por un lado, entrevistas semi-estructuradas con informantes clave (funcionarios municipales $y$ provinciales en distintas áreas de la gestión, y referentes de organizaciones no gubernamentales) y, por el otro, grupos focales con actores vinculados a la atención médica, la educación y las instituciones religiosas. Los resultados sugieren una distancia muy corta entre las ciudades en las cuales las tasas han aumentado y aquellas en las que han disminuido. La diferencia más significativa es que en Venado Tuerto se encontró una mayor fragilidad en las instituciones públicas, por la falta de articulación entre las mismas. En tanto en San Rafael, los actores entrevistados atribuyen el bajo nivel de conflictividad a una red de prevención de violencias en la cual se coordinan agencias provinciales y municipales. Sin tratarse, ni Venado Tuerto, ni San Rafael, de ciudades violentas en los niveles latinoamericanos más truculentos, ambas muestran resultados diferentes que apuntan directamente a la posibilidad de juntar a las instituciones en un entramado de conversaciones, acuerdos y políticas en conjunto.

Palabras clave Homicidio, Violencia, Mortalidad, Armas de fuego, Gobierno local, Argentina 


\section{Introducción}

Este trabajo forma parte de un estudio epidemiológico descriptivo en Argentina de la tendencia temporal de la mortalidad por homicidios en el período $1990-2007^{1}$, y la mortalidad por armas de fuego en el período 1990-2008², continuando una línea de investigación institucional en el tema ${ }^{3-5}$. La ampliación del análisis epidemiológico apelando a un abordaje cualitativo, es lo que presentaremos aquí. Es preciso aclarar que no entendemos el paso de la instancia cuantitativa a la cualitativa como si se tratara de transitar dos compartimentos estancos, territorios inconexos o lenguajes irreconciliables. Más bien lo pensamos como la conformación de un espacio heterogéneo, en el que se intenta mirar a un mismo fenómeno desde diferentes ángulos, puntos de vista capaces de entablar un cierto diálogo.

Para el análisis cualitativo se eligió como estrategia de investigación la realización de dos estudios de caso, a fin de complejizar la comprensión del fenómeno de los homicidios, produciendo información detallada sobre una realidad puntual. Las unidades de análisis elegidas fueron dos distritos (denominados en Argentina "departamentos") que en la etapa del estudio epidemiológico mostraron tendencias diferentes en relación a las tasas de homicidios por armas de fuego.

\section{Materiales y métodos}

¿Qué es exactamente un “departamento" para el derecho administrativo argentino? Después de las provincias, es decir las unidades federales autónomas que conforman la República Argentina, los departamentos constituyen la segunda forma de subdivisión territorial del país. En algunas provincias son utilizados como distritos para elegir legisladores, y también como unidades de descentralización para la administración de la justicia y de la policía. Los departamentos no coinciden necesariamente con las ciudades, o más bien con los municipios, es decir con las entidades de gobierno local. Existen departamentos que albergan un único municipio, otros que tienen un municipio y diferentes "comunas" (secciones de ese departamento que no llegan a tener rango municipal), y otros que contienen directamente varios municipios.

De hecho, los dos departamentos seleccionados presentan características administrativas diferentes. El primero fue el Departamento de General López, en Santa Fe, provincia situada en el centro del país en una región agrícola-ganadera. Es una de las provincias más importantes en términos económicos y una de las que más aporta al PBI nacional. La población total es de algo más de tres millones de habitantes, más de la mitad de los cuales se concentran en dos departamentos: Rosario, que alberga una de las ciudades más ricas del país, y la ciudad capital, que lleva el mismo nombre que la provincia; $y$, por su parte, el Departamento de General López, que tiene alrededor de doscientos mil habitantes, concentrados en las áreas urbanas de los municipios de Firmat, Rufino, Villa Cañás y, fundamentalmente, Venado Tuerto.

El segundo caso estudiado fue el Departamento de San Rafael, en Mendoza, la principal provincia de la región vitivinícola de Cuyo. A diferencia del caso anterior, en esta provincia las ciudades $-\mathrm{y}$ sus ejidos municipales - corresponden con la entidad territorial de los departamentos. Cada municipio es sede de un único departamento, subdividido en distritos o secciones. La ciudad de San Rafael es la cabecera del departamento que lleva el mismo nombre, en el cual solo existen otros distritos rurales. La población total es apenas menor que la de General López, pero en este caso la gran mayoría de los habitantes se concentran en la propia ciudad de San Rafael, mientras que los restantes distritos son pequeñas villas de montaña.

Venado Tuerto es una ciudad de algo más de ochenta mil habitantes que, en su doble condición de eje agropecuario y polo industrial, atrae a muchos trabajadores de la región sur de la Provincia de Santa Fe. Si bien no es el único municipio del departamento, lo utilizamos como referencia por ser la sede administrativa de diferentes servicios con alcance regional: el Hospital "Alejandro Gutiérrez”, el poder judicial, las líneas de emergencias médicas, los medios de comunicación y diversos establecimientos educativos, entre ellos dos subsedes de universidades nacionales. Por eso, en ciertas actividades, Venado Tuerto ejerce atracción social y cultural sobre las demás localidades del Departamento.

$\mathrm{Al}$ igual que Venado Tuerto, la ciudad de San Rafael congrega a las sedes de la administración pública del departamento, los servicios de salud y educación de mayor envergadura, los programas provinciales vinculados a la seguridad, la protección y la promoción de derechos.

Para llegar a la selección de estos dos casos utilizamos algunos criterios de exclusión que sería conveniente explicitar. En primer lugar, fueron dejados de lado los departamentos que co- 
rresponden a las capitales de las provincias argentinas y los grandes conglomerados urbanos (en particular, el Gran Buenos Aires, también llamado "Conurbano Bonaerense", un área de gran densidad poblacional que rodea a la capital del país). En segundo lugar, se excluyeron los departamentos con menos de 100.000 habitantes, porque la intención era focalizar el análisis en ciudades de mediano tamaño del interior. De esta manera, se seleccionó a Venado Tuerto por ser el departamento que presentaba mayor aumento en la tasa de homicidios por armas de fuego durante el período considerado. En el caso de San Rafael, la decisión se debió a que pertenece a una provincia en la cual varios departamentos mostraron una tendencia a la disminución de las tasas de homicidios en el período y un aumento de la variación de la proporción de causas mal definidas. Si bien San Rafael presenta más bien un estancamiento de las tasas, se lo privilegió por sobre los departamentos de Guaymallén, Las Heras, Maipú y Godoy Cruz porque todos ellos, aún mostrando una mayor disminución de sus tasas, forman parte del conglomerado urbano conocido como "Gran Mendoza", alrededor de la capital provincial. Las principales características sociodemográficas, de salud y de algunos indicadores de violencia para cada departamento al cual pertenece cada municipio pueden observarse en la Tabla 1.

La investigación cumplió todos los prerrequisitos éticos y fue aprobado por un Comité de Ética.
Los dos estudios de caso se hicieron complementando información epidemiológica cuantitativa con información cualitativa con el objetivo de ampliar el horizonte comprensivo de la problemática y ofrecer un terreno desde el cual iniciar la búsqueda de explicaciones estructurales de las tendencias temporales de la mortalidad por homicidios por armas de fuego.

Para la investigación cualitativa se hicieron grupos focales y entrevistas semi-estructuradas. El criterio de selección de los participantes surgió a partir de la hipótesis de trabajo de que el entramado institucional gubernamental y civil vinculado a las violencias, por un lado, y la lógica de puesta en práctica de las políticas vinculadas a la seguridad, por el otro, podrían diferenciarse en ambos municipios y que estas diferencias podrían ser significativas para el fenómeno estudiado. Por este motivo, se eligieron para las entrevistas tanto funcionarios y trabajadores públicos que representaban a las diversas instancias institucionales que de manera directa trabajan con la problemática de los homicidios y la violencia (directores de las áreas de acción social, violencia de género, niñez y adolescencia; gestores de la seguridad pública, policías, y la red de emergencias) como representantes de organizaciones civiles relacionadas a temas de violencia y periodistas. En total se realizaron 16 entrevistas.

Los grupos focales fueron doce (dos con maestros de escuela secundaria, dos con jóvenes religiosos, dos con jóvenes que están o estuvieron en el pasado en conflicto con la ley, dos con

Tabla 1. Información sociodemográfica y de algunos indicadores de salud y violencia en los Departamentos de San Rafael y General López.

\begin{tabular}{lcc}
\hline & $\begin{array}{c}\text { Departamento de } \\
\text { San Rafael }\end{array}$ & $\begin{array}{c}\text { Departamento de } \\
\text { General López }\end{array}$ \\
\hline Población $2001^{3}$ & 173.571 & 182.113 \\
Población $2010^{4}$ & 188.018 & 191.024 \\
Población $<1$ año $^{4}$ & 3.199 & 2.759 \\
Población $>65$ años $^{4}$ & 21.840 & 20.171 \\
Porcentaje de analfabetismo (\%) & 4,5 & 2,2 \\
Porcentaje de población con NBI $(\%)^{3}$ & 17,6 & 10,7 \\
Tasa de mortalidad general (por mil) & $7,5^{5}$ & $9,5^{6}$ \\
Tasa de Mortalidad Infantil (por mil) & 12,3 & 12,9 \\
Tasa de mortalidad por amas de fuego 1999-2002 (por cien mil) & 3,8 & 2,2 \\
Tasa de mortalidad por amas de fuego 2002-2006 (por cien mil) & 2,2 & 2,7 \\
\hline
\end{tabular}

Fuente: elaboración propia a partir de INDEC ${ }^{6,7}$, DEIS $^{8}$, Municipalidad San Rafael ${ }^{9}$, Gobierno de la Provincia de $\mathrm{Santa} \mathrm{Fe}^{10}$; y de bases de datos de mortalidad por armas de fuego de la DEIS.

Nota: El Municipio de San Rafael pertenece al Departamento de San Rafael; mientras que el Municipio de Venado Tuerto pertenece al Departamento de General López. 
familiares de jóvenes, dos con estudiantes de escuelas públicas y dos en centros de atención primaria y hospital con su personal) y para su organización contamos con la colaboración de al menos un "informante clave" por cada localidad. El criterio para elegir los participantes de los grupos focales, fue el que perteneciera a instituciones públicas u organizaciones de la sociedad civil que no estuvieran vinculadas de manera directa con las políticas e instituciones de seguridad, pero si atravesados por temas de violencia -para que opinaran sobre estas políticas e instituciones con conocimiento pero desde afuera- y que, a su vez, que no tuvieran cargos destacados o fueran líderes de las organizaciones civiles, que participaron en la investigación.

Las entrevistas y los grupos focales se llevaron adelante en los contextos de trabajo y/o estudio de los actores sociales (por ejemplo, el del centro de atención primaria se realizó en una sala del hospital municipal; el de jóvenes religiosos se concretó en la iglesia a la que asisten). Esto también fue parte de una decisión metodológica, ya que fueron entrevistados por sus roles específicos en la comunidad y se intentó comprometerlos profesional y éticamente en esa dimensión (y no por otros rasgos de sus subjetividades).

El análisis se realizó en primera instancia restituyendo las temporalidades de los actores. Para cada uno de ellos existen etapas más crudas en relación a las muertes y lesiones violentas y períodos menos trágicos. Pusimos en diálogo las convergencias y divergencias de dichas temporalidades y buscamos sus propias explicaciones (macro y micro social). Posteriormente, desmenuzamos el sentido de un acto violento, las causantes que identificaron, la dirección sugerida (hacia quienes se ejerce violencia). Y finalmente nos dedicamos al análisis de la estructura gubernamental para paliar (con políticas de prevención) y para atender situaciones de violencia.

Todos estos actores fueron esenciales para reconstruir la lógica de las prácticas sociales y representaciones vinculadas a la violencia, el uso de armas de fuego y la mortalidad por homicidios.

Los métodos cualitativos elegidos nos permitieron una mirada más profunda de las prácticas y contextos de las políticas aplicadas a la seguridad, con sus insuficiencias y contradicciones, en los lugares seleccionados, evitando caer en la ilusión de los organigramas o la ingenuidad de lo normativo.

\section{Resultados}

Tanto en Venado Tuerto como en San Rafael, el mapa de instituciones y actores entrevistados resultó de una negociación entre el plan original de entrevistas y lo que durante el trabajo de campo resultó factible. En tal sentido, cabe destacar que mientras en el departamento santafecino elegimos una ONG representativa en el terreno de atención a casos de violencia, entre otras tantas existentes, en San Rafael la incidencia de las organizaciones de la sociedad civil en la trama de las políticas de prevención de violencias es muy limitada. Algo parecido puede decirse de las iglesias (inclusive las evangélicas, que suelen ser más activas en la atención de estos problemas), las cuales se limitan a discretos programas de rehabilitación de adictos. Ahora bien, eso no significa que las políticas de prevención y atención ante casos de violencia estén más débilmente articuladas en el departamento mendocino. Al contrario, los mismos actores entrevistados (policías, profesionales de escuelas, responsables de las emergencias médicas, etc.) atribuyen el bajo nivel de conflictividad de la ciudad a una red de prevención de violencias en la cual se coordinan agencias provinciales y municipales.

Las entrevistas en Venado Tuerto se realizaron en plena campaña política por las elecciones de 2011 y la "cuestión de la seguridad" ocupaba un cierto espacio en la puja proselitista, aunque sensiblemente menor al que se registra en los distritos metropolitanos del Gran Buenos Aires. No faltaban, de todos modos, candidatos que agitaran las banderas del combate al delito proponiendo como uno de los puntos básicos del programa la producción de un "mapa de la inseguridad”. Una de las fuerzas políticas opositoras, desfilaba en vehículos que por altoparlantes difundían un spot publicitario proclamando: "vamos por el cambio, para generar una política municipal de seguridad que dé tranquilidad a nuestros vecinos".

No es casual que la oposición al actual gobierno comunal santafecino utilice esta idea. En efecto, el rol de la municipalidad en la elaboración de políticas de seguridad conduce de lleno a uno de los principales dilemas que atraviesan las instituciones y los actores que, más o menos directamente, trabajan en la atención de las situaciones locales de violencia. En la entrevista, el Intendente de Venado Tuerto reconoce la existencia de una desconexión entre el sistema judicial-policial (que constitucionalmente responde al gobierno provincial) y el municipio, que en cuestio- 
nes de delitos "ni siquiera opina”. La policía depende de un jefe que tiene su sede en la capital de la Provincia de Santa Fe, localizada a 400 kilómetros de Venado Tuerto y, según considera el Intendente, la lógica verticalista que opera en esta institución impide la coordinación de políticas en diálogo con el municipio.

El caso de San Rafael presenta algunas características diferentes. La policía provincial, por ejemplo, no sólo está repartida en las comisarías seccionales como en cualquier otra ciudad, sino que también posee órganos específicos que buscan una mejor articulación con otros organismos de gobierno. El Ministerio de Seguridad de la Provincia de Mendoza instaló en la ciudad una de las oficinas de la Policía Comunitaria, que atiende a San Rafael y a dos departamentos contiguos de menor población (Malargüe y General Alvear). Esta dependencia policial tiene un programa de asistencia a las víctimas con objetivos muy amplios, pero en la práctica - según el relato de un agente policial y de una gestora en seguridad que trabajan en la oficina - se dedica casi exclusivamente a la contención de víctimas de abuso sexual.

Por otra parte, desde fines de 2010 se puso en funcionamiento una Comisaría del Menor, lo cual implicó una separación del tratamiento de "menores en conflicto con la ley" de las comisarías de seguridad, donde los niños solían compartir los espacios de detención con los adultos. La entrevista con el titular de esta nueva comisaría fue interrumpida por la detención de un menor que había herido a alguien con un arma de fuego. Al despacho del comisario entraron varias veces agentes policiales que consultaban sobre el procedimiento, por lo cual pudimos observar la forma en que articulan con el poder judicial y el Órgano Administrativo Local (OAL), creado en la provincia para poner en práctica la Ley Nacional 26.061 de Protección Integral de Derechos de Niñas, Niños y Adolescentes (2005). Muchas veces los menores son derivados desde diferentes centros de salud y el propósito de la Comisaría consiste en acelerar la vía judicial del caso.

La policía provincial mendocina instaló una línea de emergencias gratuita (102), que absorbe todo tipo de llamados, inclusive denuncias anónimas por maltratos, que son derivadas a la red que coordina el OAL. Cuando el 102 recibe una denuncia avisa a la Policía Comunitaria (si el caso es grave y requiere de agentes policiales para acercarse al lugar) o bien se cita a las personas denunciadas a través de los centros de salud, cuando la denuncia procede de una localidad más alejada de San Rafael.
Las entrevistas con los funcionarios de la Policía Comunitaria, de la Comisaría del Menor y del OAL muestran un diagnóstico común sobre la centralidad de la violencia intrafamiliar en el caudal de casos atendidos por estas instituciones. Eso parece explicarse por la consolidación de espacios institucionales que facilitan la canalización de denuncias y las viabilizan en un sistema único, evitando que la víctima - como sucede en Venado Tuerto - tenga que repetir varias veces el mismo relato ante diferentes autoridades. En San Rafael, las víctimas pueden utilizar una línea telefónica 102 para denunciar un caso de violencia, o pueden dirigirse directamente a la Policía Comunitaria.

Durante una de las semanas en que realizamos el trabajo de campo en la ciudad de San Rafael, hubo en las oficinas del OAL una reunión para establecer un protocolo de atención a casos de abuso sexual de menores, al que asistieron representantes de la Comisaría del Menor y la Policía Comunitaria, juzgados correccionales, asistentes sociales del Hospital e inspectores de escuelas. Aunque no pudimos presenciar la reunión, reconstruimos por las entrevistas posteriores que se discutieron medidas para solucionar falencias de esta red de atención a casos de violencia doméstica, como por ejemplo la dificultad de llegar a las zonas rurales más alejadas de la ciudad. Estas reuniones se realizan desde la creación de un "Programa de Prevención y Atención Integral del Maltrato a la Niñez, Adolescencia y Familia" (1998), pionero a nivel nacional. En este entramado de actores, el OAL cumple una función central, porque se encarga de distribuir las denuncias recibidas por distintas instituciones: los gabinetes psicopedagógicos de las escuelas, los consultorios externos de los centros de salud y, obviamente, las denuncias hechas directamente por las víctimas a las líneas telefónicas.

Los grupos focales con profesionales de escuelas públicas y con trabajadores del principal hospital de San Rafael, reafirman la centralidad de esta red de acción conjunta. En el Hospital Provincial Teodoro Schestakow, por ejemplo, entrevistamos un grupo de profesionales que atienden casos de violencia. Además de recibir las denuncias directas a través de la línea 102, también se ocupan de conflictos que detectan directamente de los servicios del hospital: guardia, internación, psiquiatría, etc. En estos casos, los médicos actúan de oficio, fundamentalmente cuando identifican una situación de violencia o maltrato en menores de 17 años. El área de atención de violencias está integrada por un médico, una psicóloga y un trabajador social que traba- 
jan dentro del Hospital, pero también tienen un equipo que se desplaza en automóvil por la ciudad para atender algunas denuncias y situaciones puntuales de maltrato en los hogares.

En este sentido se percibe una diferencia con Venado Tuerto, donde el problema de la desarticulación en la red pública de asistencia a los casos de violencia no se reduce a la falta de diálogo entre policía y municipalidad, sino que se alcanza a todas las instituciones que directa o indirectamente se ocupan de la prevención de la violencia, así como de la atención ante sus eventuales efectos en los habitantes. En Venado Tuerto el gobierno municipal desestimó la estrategia de creación de una policía local o "guardia urbana", como se hizo en otras ciudades de esta misma provincia, y optó por el desarrollo de políticas preventivas de dos tipos: programas sociales apuntados a la familia, la niñez y la adolescencia, por un lado; y estrategias situacionales de intervención sobre el espacio urbano (limpieza de terrenos baldíos, iluminación de la ciudad, etc.).

La Secretaría de Acción Social del Municipio de Venado Tuerto creó en el año 2008 un Programa de Género y Familia, que quedó a cargo de una trabajadora social, una psicóloga y una pasante de abogacía para el asesoramiento legal. El equipo trabaja en los barrios a través de grupos interdisciplinarios distribuidos en los centros de salud, porque consideran que las mujeres se acercan con mayor facilidad a los médicos para realizar consultas que pueden derivar en la atención a un caso de violencia doméstica. Además, llevan adelante actividades de promoción en escuelas e iglesias, donde dan cursos de promotores comunitarios para trabajar con violencia de género. Lo mismo sucede con el área de Niñez y Juventud que, bajo la órbita de la misma secretaría, se distribuye territorialmente en torno a los centros de salud.

Desde la reforma de la Constitución Nacional en 1994, y como consecuencia de la ratificación del rango constitucional de la Convención de los Derechos del Niño, se amplió considerablemente el marco legal para la protección de estos derechos, tanto a nivel nacional como en la Provincia de Santa Fe (en el caso de la Provincia de Santa Fe, nos referimos a la Ley Provincial de Violencia Familiar No 11.529 de 1997 y más recientemente la Ley Provincial de Promoción y Protección Integral de los Derechos de las Niñas, Niños y Adolescentes, $\mathrm{N}^{\circ} 12.967$ de 2009). De las entrevistas con encargados de las áreas de género, familia y niñez, $\mathrm{y}$ en los grupos focales realizados con los integrantes de otras instituciones que trabajan en dis- tintos niveles de atención ante casos de violencia, aparece reiteradamente un reclamo por la grieta que se abre entre las demandas que producen las nuevas leyes y los recursos materiales para cumplir con los procedimientos legales.

Actualmente, por ejemplo, las leyes en vigencia establecen que ante casos de abuso de menores se debe tomar la denuncia directamente al niño, pero la policía santafecina muchas veces se niega a hacerlo y cuando no existe la alternativa de dejar al menor en cuidado de otro pariente, las medidas excepcionales para separarlo del entorno familiar se hacen difíciles sin la presencia de Juzgados de Menores para el seguimiento del caso. Algo parecido sucede en las situaciones de violencia de género, donde además de la resistencia de la policía a la hora de tomar las denuncias, existen obstáculos todavía más agudos a la hora del acompañamiento de las víctimas. La municipalidad sostiene programas para la prevención de la violencia contra las mujeres, pero una vez que ellas traspasan el delicado umbral de la denuncia, son pocas las instituciones que pueden brindarle apoyo. En suma, la trama de atención a casos de violencia en Venado Tuerto, a diferencia de San Rafael, está signada por la des-coordinación de los actores involucrados en estos procesos.

La precariedad del archipiélago institucional abocado a la prevención de violencias en Venado Tuerto puede entenderse a través de la reconstrucción hipotética del itinerario recorrido por una mujer que llega al Hospital Regional con lesiones, como explicaron los profesionales de la salud en la charla del grupo focal. Una de las principales vías para canalizar el seguimiento de una denuncia de violencia de género es una ONG llamada "Línea Amarilla". Se trata de una organización que existe hace dieciséis años, en la que todos sus integrantes trabajan en forma voluntaria ad honorem a través de una línea telefónica que asiste a personas "en crisis" (fundamentalmente violencia de género e intentos de suicidio), brindando asistencia psicológica y, si fuera necesario, asesoramiento legal.

Aunque esta ONG es una referencia en el área, las condiciones materiales en las que trabaja son muy precarias. Cuando visitamos la casa en que funcionan, recientemente habían estado casi un mes sin atender llamadas porque cuentan con un único número no rotativo y en la zona de la sede robaron el cableado telefónico. Sin embargo, la principal dificultad manifestada durante la entrevista por la presidenta (psicóloga) y la secretaria (trabajadora social), apuntó a los obstáculos que ponen la policía y los juzgados para 
atender a las víctimas. Las entrevistadas aseguraron que la desatención y hasta la victimización de las mujeres que se acercan a denunciar violencias de género, han llegado a desencadenar homicidios perpetrados como represalia por la denuncia cuando las víctimas regresan a sus casas. Inclusive, la presidenta de Línea Amarilla fue amenazada de muerte varias veces y en una oportunidad un hombre intentó atropellarla con su camioneta para amedrentarla, por su ayuda a las mujeres en el seguimientos de estos casos.

En Venado Tuerto, uno de los principales aliados de Línea Amarilla para compensar la desatención de las instituciones públicas es, según contaron, la prensa local. Entrevistamos a la periodista de un diario encargada de la sección de policiales, a quienes las integrantes de la ONG reconocieron como una aliada con la que contaban para denunciar casos de violencia. La periodista reconoció que varias instituciones, y en algunos casos víctimas particulares, le acercan información para ejercer presión sobre las agencias estatales y facilitar intervenciones que de otra forma nunca se iniciarían. Por otra parte, desde la visión de los periodistas locales, es decir desde el registro cotidiano de hechos policiales, además de la violencia doméstica existe un incremento de los robos a mano armada, que ellos vinculan al consumo de drogas entre los jóvenes. Un fenómeno que, pese a las diferencias en las tramas institucionales y en el nivel de coordinación de los actores, se repite en ambos departamentos.

En Venado Tuerto, la percepción del uso de armas cortantes y armas de fuego aparece inmediatamente asociada a los jóvenes, en particular a las riñas que se multiplican los fines de semana. El consumo de drogas y la ingesta de alcohol aparecieron con especial fuerza en las entrevistas, relacionadas a los intentos de suicidio y a los accidentes de tránsito. En San Rafael, junto al grupo focal con los profesionales del Hospital, realizamos una entrevista al jefe del área de emergencias. La percepción que ambos grupos tienen es que en los últimos años el consumo de drogas incide en los casos que atienden, violencias interpersonales, y accidentes de tránsito.

En contraste, los casos de muerte por armas de fuego aparecen con mayor dificultad en la memoria de los entrevistados. Cuando se indaga explícitamente sobre el tema, solamente los policías en Venado Tuerto pusieron cierto énfasis sobre casos vinculados a riñas entre jóvenes y las encargadas de Línea Amarilla mencionaron la existencia de algunos femicidios. Otros aseguran que recuerdan únicamente hechos de sangre pro- vocados por armas cortantes. Tanto si recuerdan casos de muertes por violencias o no, todos los entrevistados coinciden en percibir un incremento de los niveles de violencia interpersonal y se manifiestan escépticos en relación a la eficacia de las instituciones públicas.

En San Rafael, solamente el jefe del servicio de emergencias del Hospital Schestakow, que trabaja en ese lugar desde 1994, asegura que cuando comenzó la guardia recibía aproximadamente dos heridos de bala por mes. Aunque no lleva estadísticas precisas, sostiene que hubo un aumento importante de los casos de violencia durante los fines de semana, y que en particular están recibiendo cada sábado una o dos personas heridas de bala o armas blancas. A su vez, pese a que no es su función indagar los motivos por los cuales alguien llega herido a la guardia, desde su punto de vista la mayor parte de los casos son provocados en peleas en un contexto de consumo de alcohol y drogas, mientras que la incidencia de las heridas por robos no constituye un caudal importante de casos. La mayor parte de los actores entrevistados tienen una percepción muy lejana del riesgo de ser víctimas de hurtos, robos o agresiones físicas en los espacios públicos. Eso quedó claro en los grupos focales con jóvenes de escuelas públicas y con jóvenes religiosos. No sólo ninguno temía ser víctima de un delito en la ciudad, sino que además ninguno había pasado siquiera por un intento de asalto. "San Rafael, como ven, es una ciudad segura" o "acá no ves las cosas que pasan en la tele de Buenos Aires": son frases recurrentes entre estos adolescentes. Una visión parecida dieron las asistentes sociales de escuelas medias, que trabajan rotando en varios establecimientos educativos realizando orientación pedagógica para mejorar el rendimiento escolar. Nuevamente, cuando se indaga sobre la conflictividad, desde la visión de las escuelas la preocupación casi excluyente es la violencia intrafamiliar, tanto por los casos de menores abusados como por los de "alumnos problemáticos" que según las asistentes sociales trasladan a la escuela un maltrato que viven en sus hogares.

De todos modos, las profesionales no recuerdan ningún caso de violencia en una escuela de San Rafael que haya involucrado la presencia de armas de fuego. Quizás los únicos dos entrevistados que brindaron una visión algo diferente fueron el titular de una de las comisarías y el director del principal diario de la ciudad. El comisario se mostró alarmado con las agresiones físicas entre personas que a veces comienzan con insultos y terminan en lesiones graves. Para el 
policía, esto se debe al crecimiento de la marginalidad en la periferia de la ciudad (donde dice que se concentran la mayor parte de los casos) y a la blandura del poder judicial a la hora de castigarlos. Por su parte, el director del diario considera que existe en San Rafael, como en todo el país, un crecimiento de la "inseguridad". Define este fenómeno por la presencia cada vez más clara de delitos contra la propiedad en los que se utilizan armas de fuego y cortantes, algo que atribuye sin dudarlo a los recién llegados a la ciudad.

Este contraste entre la mayor parte de los entrevistados y estos dos últimos, puede explicarse tal vez por la naturaleza del trabajo que realizan, puesto que reciben directamente - ya sea para intervenir o para noticiar - gran parte de los hechos delictivos que suceden. Sin embargo, llama la atención que cuando pasamos a una interrogación sobre casos concretos, tanto el comisario como el periodista vacilaban, recordaban pocos hechos con armas de fuego, no eran claros en las ejemplificaciones. Es probable, entonces, que la diferencia de opiniones se nutra también de discrepancias con las políticas públicas, si no directamente con los partidos políticos que las llevan adelante, y con visiones muy arraigadas en los oficios a los que pertenecen, como la disputa histórica de la policía con el poder judicial, o la tendencia de la prensa gráfica a construir relatos sobredimensionados sobre el delito en las ciudades.

En contraste, todos los entrevistados de Venado Tuerto coincidieron en indicar al departamento santafecino como un territorio inseguro. Los relatos repiten tres tópicos que parecen tener una gran eficacia como modo de interpretación del presente de la ciudad. Uno es el grado de exposición de las desigualdades sociales, muy marcadas por ser una región agrícola ganadera donde las ganancias tienden a concentrarse en pocas familias. "Acá la opulencia se muestra", sintetiza un periodista, habituado a que los cambios en el paisaje local se traduzcan más en el paseo de coches suntuosos por las calles céntricas que en mejoras de infraestructura en los espacios públicos.

El segundo tópico es una lectura nostálgica acerca de un pasado difuso en su periodización, pero omnipresente en las narraciones sobre la historia reciente de la ciudad: muchos habitantes sostienen que aumentaron significativamente los delitos, otros opinan que no aumentaron pero que ciertos hechos - en particular los robos callejeros - se hicieron más violentos. Según estos relatos, el supuesto desmadre de las normas más elementales de convivencia se pone de manifiesto en noches de los fines de semana pobladas de consumo de drogas y riñas entre jóvenes. Todos los entrevistados acuerdan en que las cosas ya no son lo que eran y, más precisamente, que están mucho peor que antes. Sin embargo, si intentamos indagar cuándo se produce el quiebre, en qué momento las cosas cambiaron o inclusive cuándo estaban mejor, la memoria de los entrevistados se torna más borrosa.

Por último, el proceso de recuperación económica de la región en los últimos años, estimulado por el boom en las exportaciones de soja, provocó la llegada de trabajadores de otras localidades. En las entrevistas aparece un tercer tópico que asocia la violencia con la presencia de estos nuevos vecinos. La dinámica entre los "establecidos" y los “extraños”, estudiada por Elias y Scotson $^{11}$ en una pequeña localidad inglesa, se constata aquí en numerosas referencias a una desconfianza depositada sobre inmigrantes provenientes de otras ciudades del país, e inclusive de países limítrofes. Aunque no fueron los únicos en mencionarlo, los policías entrevistados recordaron especialmente los asesinatos de un agente policial que resistió un asalto a una mutual en 2007 y de un carnicero durante un robo a mano armada, al año siguiente, donde los acusados provenían de otras ciudades.

Es preciso señalar que controlamos estos casos en los medios gráficos locales, y el lugar de residencia de los acusados no resulta tan claro. En el caso del asesinato de 2007, el acusado - condenado a 25 años de prisión - era proveniente de Río Cuarto (Provincia de Córdoba), pero se habría instalado en Santa Fe con su madre a los 15 años. En el caso de 2008, los ladrones eran dos: uno era de Venado Tuerto y fue condenado a 20 años de prisión por ser el responsable del disparo que provocó la muerte al carnicero, el otro era de Junín (Provincia de Buenos Aires) y fue condenado a 11 años de prisión por complicidad.

\section{Conclusiones}

Este estudio se propuso comparar dos departamentos del interior de la Argentina que mostraban tendencias diferentes en relación a las tasas de homicidios por armas de fuego. No obstante, los estudios de caso realizados sugieren una distancia muy corta entre las ciudades en las cuales las tasas han aumentado y aquellas en las que han disminuido o se han mantenido estables, en torno a cifras muy bajas si se las compara con ciudades del interior en otros países latinoamericanos. 
Si cambiamos la escala de observación, comparando estos casos con ciudades de similar tamaño en Brasil, Colombia o México, es preciso reconocer que en ambas ciudades argentinas las muertes por violencias, incluyendo las situaciones de violencia con armas de fuego, no tienen una presencia fuerte en la percepción que los entrevistados manifiestan de su vida cotidiana en la ciudad.

Raramente un entrevistado habló espontáneamente de hechos de violencia con armas de fuego y cuando se realizó alguna pregunta sobre situaciones de violencia en general, podríamos concluir que los actores se separaron en dos grupos. En primer lugar, aquellos que trabajan para paliar los efectos de la violencia, a través de intervenciones reactivas (servicios de emergencias médicas, líneas telefónicas, organizaciones de la sociedad civil e instituciones públicas), centraron la atención en los accidentes de tránsito, especialmente los que involucran a motociclistas. En segundo lugar, los entrevistados que trabajan en políticas preventivas (programas gubernamentales, iglesias, establecimientos educativos) identifican como núcleo problemático la violencia intrafamiliar. Mientras el fenómeno de los accidentes con motos suele ser explicado en el contexto de un aumento significativo de estos vehículos provocado por las facilidades de financiamiento, la violencia de género y los abusos sexuales a menores de edad parecen haber ingresado, en las provincias del interior del país, en una etapa de mayor visibilización de los casos, por la existencia de normativa e instituciones que promueven activamente la realización de denuncias.

Otra coincidencia entre ambas ciudades es la percepción del consumo de drogas como un fenómeno difícil de frenar entre los jóvenes, que acarrea consecuencias en el campo de la violencia interpersonal. Pero no debe pasarse por alto la distancia que existió en las entrevistas y grupos focales, entre la definición en abstracto de ciertos problemas y la indagación de casos concretos que permitirían explicarlos. La desestructuración de la familia, el impacto de la drogadicción en los jóvenes, los problemas que todo eso arrastra en las instituciones escolares: es preciso reconocer se trata de cuestiones que también operan en un nivel cultural, donde la nostalgia, la sensación de un pasado mejor que se ha perdido, tiene un costado de construcción mítica, bastante efectiva a la hora de producir relatos urbanos. De este modo, cuando el entrevistador pasa de las grandes definiciones ("violencia juvenil", "crisis esco- lar", "familias conflictivas"), manejadas por los propios actores, a una indagación de casos puntuales, el problema se vuelve más difuso y no es extraño que los casos mencionados muestren una cara menos dramática de la que podía presumirse por los rótulos.

Finalmente, más allá de los puntos en común que permiten relativizar la distancia entre ambas ciudades, hay que considerar algunas diferencias. La más significativa es que en Venado Tuerto se encontró una mayor fragilidad en las instituciones públicas, no sólo por la inexistencia de organismos que vimos funcionar en San Rafael (Juzgados de menores y de familias, comisaría del menor, policía comunitaria, un organismo específico para coordinar políticas contra la violencia familiar, etc.), sino también por la falta de articulación entre las instituciones existentes.

En San Rafael, los mismos actores entrevistados (policías, profesionales de escuelas, responsables de las emergencias médicas, etc.) atribuyen el bajo nivel de conflictividad de la ciudad a una red de prevención de violencias en la cual se coordinan agencias provinciales y municipales. Lo cual explicita las diferentes articulaciones halladas entre lo local, departamental y provincial en ambos municipios, que pone de relieve la baja eficacia del "gobierno a distancia" 12 en el tema de la seguridad que presupone una alta política provincial y una baja política municipal, lógica dominante en buena parte del territorio nacional y señalada en Venado Tuerto. Otro problema encontrado fue la débil y/o compleja relación y articulación entre el sistema judicial y la policía. Todo ello nos lleva a afirmar esa des-coordinación que señalamos y que no es propia de las situaciones abordadas sino una característica común no solo en Argentina, sino también en muchos otros países de América Latina.

Allí donde no llega ni el gobierno provincial ni el municipal, en Venado Tuerto se instalan organizaciones de la sociedad civil y grupos religiosos que trabajan para contener situaciones conflictivas ya desencadenadas, muchas de las cuales en San Rafael se evitan fácilmente con una mínima coordinación entre instituciones públicas. Sin tratarse, ni Venado Tuerto, ni San Rafael, de ciudades violentas en los niveles latinoamericanos más truculentos, ambas muestran resultados diferentes en la prevención de violencias que apuntan directamente a la posibilidad de juntar a las instituciones en un entramado de conversaciones, acuerdos y políticas en conjunto. 


\section{Colaboradores}

H Spinelli: trabajó en la concepción del estudio, redacción final y formato del artículo. M Alazraqui: trabajó en la concepción del estudio y en la redacción final. D Galeano: participó en el trabajo de campo, análisis e interpretación de los datos y en la redacción final. S Calandrón: participó en el trabajo de campo, análisis e interpretación de los datos.

\section{Referencias}

1. Spinelli H, Alazraqui M, Zunino G, Pérez E. Estudio multi-céntrico de la mortalidad por homicidios en América Latina: Argentina, Brasil, Colombia y México. Informe Argentina. Buenos Aires; 2010. Mimeo

2. Spinelli H, Zunino G, Alazraqui M, Guevel C, Darraidou V. Mortalidad por armas de fuego en la Argentina, 1990-2008. Buenos Aires: Organización Panamericana de la Salud; 2011.

3. Spinelli H, Macías G, Darraidou V. Procesos macroeconómicos y homicidios. Un estudio ecológico en los partidos del Gran Buenos Aires (Argentina) entre los años 1989 y 2006. Salud Colectiva 2008; 4(3):283-299.

4. Zunino MG, Souza ER, Lauritzen B. Estudio epidemiológico comparativo de la mortalidad por armas de fuego en Brasil y Argentina, 1990-2005. Salud Colectiva 2008; 4(3):349-361.

5. Zunino MG, Spinelli H, Alazraqui M. Muertes por Armas de Fuego: Un Eclipse en los Sistemas de Información en Salud. Salud Colectiva 2006; 2(3): 259-267.

6. Instituto Nacional de Estadísticas y Censos (INDEC). Censo Nacional de Población, Hogares y Viviendas. 2001. [Internet]. [acceso 2012 feb 16]. Disponible en: http://www.indec.gov.ar/webcenso/ provincias_2/provincias.asp

7. Instituto Nacional de Estadísticas y Censos (IN DEC). Censo Nacional de Población, Hogares y Viviendas. 2010. [Internet]. [acceso 2012 feb 16]. Disponible en: http://www.censo2010.indec.gov.ar

8. Dirección de Estadísticas e Información en Salud Boletín 129. Natalidad, Mortalidad General, Infantil y Materna por lugar de residencia. Argentina. Año 2009. [acceso 2012 feb 16]. Disponible en: http://www.deis.gov.ar/publicaciones/archivos/ Boletin 129.pdf

9. Sistema Estadístico municipal. Municipalidad de San Rafael. 2001 [Internet] [acceso 2012 feb 16]. Disponible en: http://www.sanrafael.gov.ar/estadisticas/ social.pdf

10. Análisis de situación de salud de la población de la Provincia de Santa Fe. Primer informe. 2011 [Internet] [acceso 2012 feb 16]. Disponible en: http://www. santafe.gov.ar/index.php/web/content/download/ 128722/636232/file/02\%20Primer\%20Informe\% 20sala\%20de\%20situacion\%20para\%20web.pdf

11. Elias N, Scotson J. Os estabelecidos e os "outsiders": sociologia das relações de poder a partir de uma pequena comunidade. Rio de Janeiro: Jorge Zahar; 1994.

12. Saín M. El leviatán azul: policía y política en la Argentina. Buenos Aires: Siglo XXI; 2008. 